\title{
Salts and solvents effect on the crystal structure of imidazolium dicarboxylate salt based coordination
}

\section{networks.}

Pierre Farger, ${ }^{a}$ Cédric Leuvrey, ${ }^{a}$ Guillaume Rogez, ${ }^{a}$ Michel François, ${ }^{b}$ Pierre Rabu, ${ }^{a}$ Emilie Delahaye $e^{a *}$

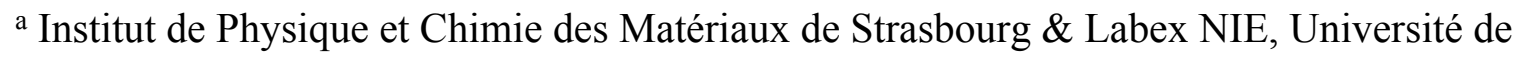
Strasbourg, CNRS UMR 7504, 67034 Strasbourg Cedex 2, France.

b Institut Jean Lamour, CNRS and Université de Lorraine, BP 70239, 54506 Vandœuvre-lesNancy, France.

Present Adress: ${ }^{\#}$ CNRS, Laboratoire de Chimie de Coordination, 205 route de Narbonne, BP 44099, 31077 Toulouse Cedex 4, France and Université de Toulouse, UPS, INPT, 31077 Toulouse Cedex 4, France.

*Corresponding author:

Emilie Delahaye

E-mail: emilie.delahaye@1cc-toulouse.fr 


\section{$\mathrm{X}$ ray diffraction}

Table S1. Selected angles for the compounds 1, 3, 5 .

\begin{tabular}{llllll}
\hline \multicolumn{1}{c}{1} & \multicolumn{2}{c}{5} \\
\hline O2-Ni1-O5 & $92.7(3)$ & O1-Ni1-O8 & $91.54(11)$ & O2-Ni1-O7 & $92.14(9)$ \\
O5-Ni1-O6 & $88.8(3)$ & O8-Ni1-O5 & $90.67(11)$ & O7-Ni1-O6 & $82.60(9)$ \\
O6-Ni1-O7 & $91.3(3)$ & O5-Ni1-O6 & $91.88(10)$ & O6-Ni1-O5 & $93.56(8)$ \\
O7-Ni1-O2 & $87.2(3)$ & O6-Ni1-O1 & $86.13(10)$ & O5-Ni1-O2 & $91.65(8)$ \\
O2-Ni1-O4 & $87.2(2)$ & O1-Ni1-O3 & $92.10(9)$ & O2-Ni1-O1 & $95.71(10)$ \\
O8-Ni1-O4 & $177.7(3)$ & O3-Ni1-O7 & $175.71(9)$ & O1-Ni1-O3 & $170.06(10)$ \\
\hline
\end{tabular}

Table S2. Selected bonds for the compounds 1, 3, 5 .

\begin{tabular}{lcllll}
\hline \multicolumn{2}{c}{1} & \multicolumn{2}{c}{$\mathbf{5}$} \\
\hline Ni1-O5 & $2.043(7)$ & Ni1-O1 & $2.072(2)$ & Ni1-O1 & $2.041(2)$ \\
Ni1-O4 & $2.057(7)$ & Ni1-O3 & $2.086(2)$ & Ni1-O2 & $2.017(2)$ \\
Ni1-O2 & $2.059(7)$ & Ni1-O5 & $2.094(2)$ & Ni1-O3 & $2.093(2)$ \\
Ni1-O7 & $2.065(5)$ & Ni1-O6 & $2.066(3)$ & Ni1-O5 & $2.0743(18)$ \\
Ni1-O6 & $2.081(7)$ & Ni1-O7 & $2.095(2)$ & Ni1-O6 & $2.059(2)$ \\
Ni1-O8 & $2.084(7)$ & Ni1-O8 & $2.041(3)$ & Ni1-O7 & $2.048(2)$ \\
Ni-O $_{a v}$ & 2.065 & Ni-O & 2.076 & Ni-O & 2.055 \\
\hline
\end{tabular}

Table S3. Selected angles for the compounds 2, 4, 6.

\begin{tabular}{llllll}
\hline \multicolumn{2}{c}{2} & \multicolumn{2}{c}{6} \\
\hline O2-Co1-O4 & $91.9(3)$ & O1-Co1-O5 & $91.53(8)$ & O1-Co1-O2 & $93.14(5)$ \\
O4-Co1-O6 & $91.3(3)$ & O5-Co1-O7 & $91.58(8)$ & O2-Co1-O3 & $92.20(5)$ \\
O6-Co1-O8 & $86.3(2)$ & O7-Co1-O6 & $92.74(8)$ & O3-Co1-O7 & $93.46(6)$ \\
O8-Co1-O2 & $90.4(3)$ & O6-Co1-O1 & $84.58(7)$ & O7-Co1-O1 & $81.14(5)$ \\
O2-Co1-O7 & $85.9(3)$ & O1-Co1-O3 & $95.22(7)$ & O1-Co1-O6 & $86.82(7)$ \\
O7-Co1-O5 & $177.6(3)$ & O3-Co1-O8 & $174.17(6)$ & O6-Co1-O4 & $169.26(6)$ \\
\hline
\end{tabular}


Table S4. Selected bonds for the compounds 2, 4, 6 .

\begin{tabular}{lccccc}
\hline \multicolumn{2}{c}{2} & \multicolumn{2}{c}{$\mathbf{6}$} \\
\hline Co1-O5 & $2.053(8)$ & Co1-O1 & $2.1022(16)$ & Co1-O1 & $2.0855(17)$ \\
Co1-O2 & $2.080(6)$ & Co1-O3 & $2.1258(15)$ & Co1-O2 & $2.1326(12)$ \\
Co1-O4 & $2.083(7)$ & Co1-O5 & $2.054(2)$ & Co1-O3 & $2.0469(14)$ \\
Co1-O7 & $2.098(7)$ & Co1-O6 & $2.099(2)$ & Co1-O4 & $2.1270(15)$ \\
Co1-O6 & $2.128(7)$ & Co1-O7 & $2.1310(17)$ & Co1-O6 & $2.0808(15)$ \\
Co1-O8 & $2.127(6)$ & Co1-O8 & $2.1416(17)$ & Co1-O7 & $2.0849(13)$ \\
Co-O $_{\mathrm{av}}$ & 2.095 & Co-O & 2.109 & Co-O & 2.093 \\
\hline
\end{tabular}



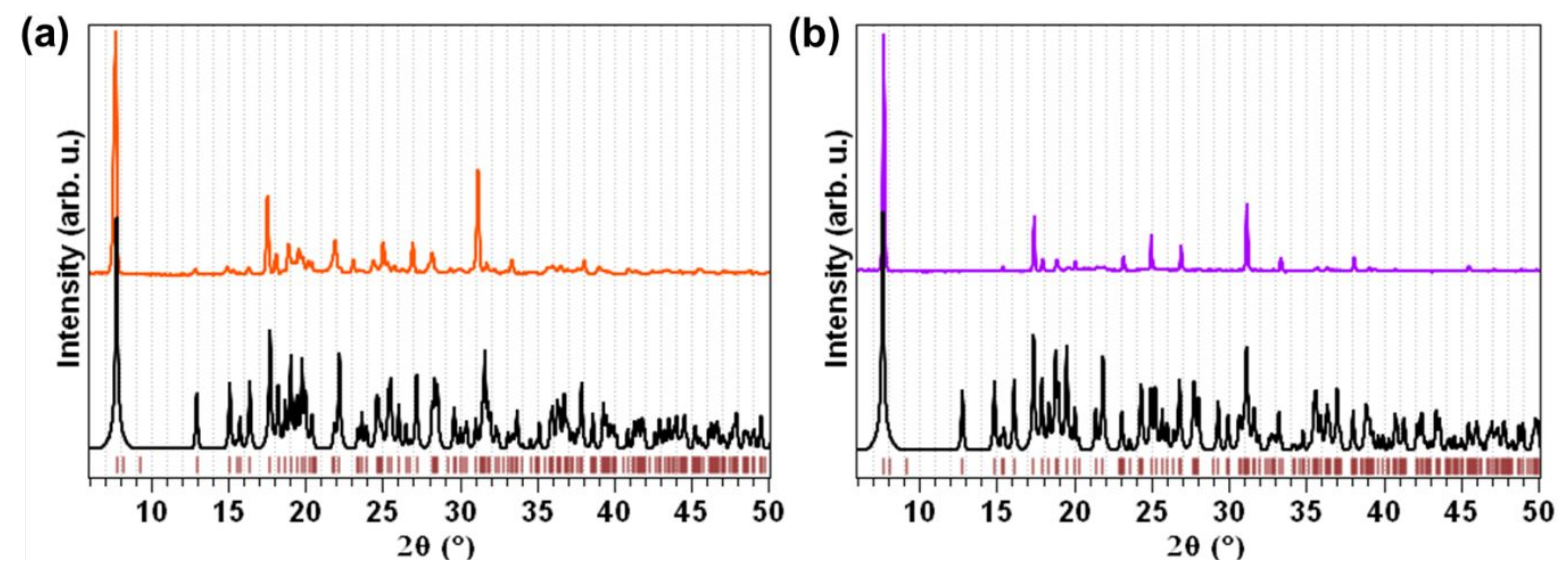

Figure S1. Comparison of the experimental powder X-ray diffraction pattern (a) for 1 (orange line) and (b) for $\mathbf{2}$ (purple line) with the calculated pattern from single crystals X-ray data (black line). The brown vertical lines indicate the position of the calculated diffraction lines.
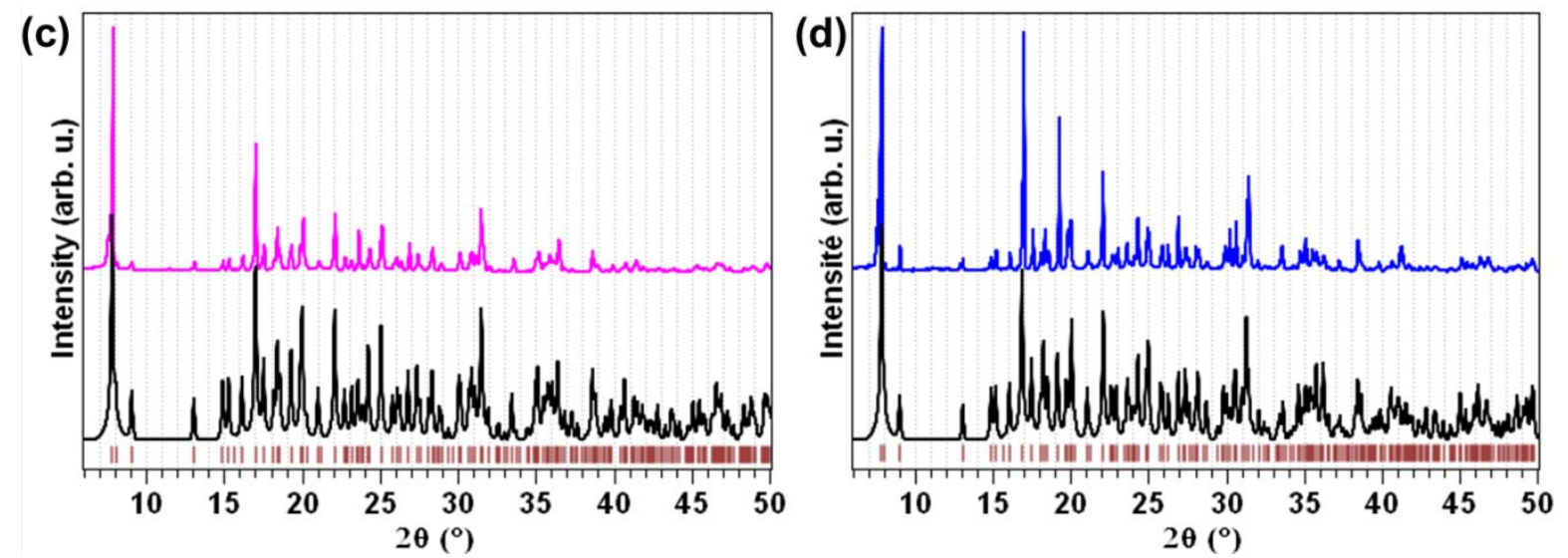

Figure S2. Comparison of the experimental powder X-ray diffraction pattern (a) for 3 (pink line) and (b) for 4 (blue line) with the calculated pattern from single crystals X-ray data (black line). The brown vertical lines indicate the position of the calculated diffraction lines.
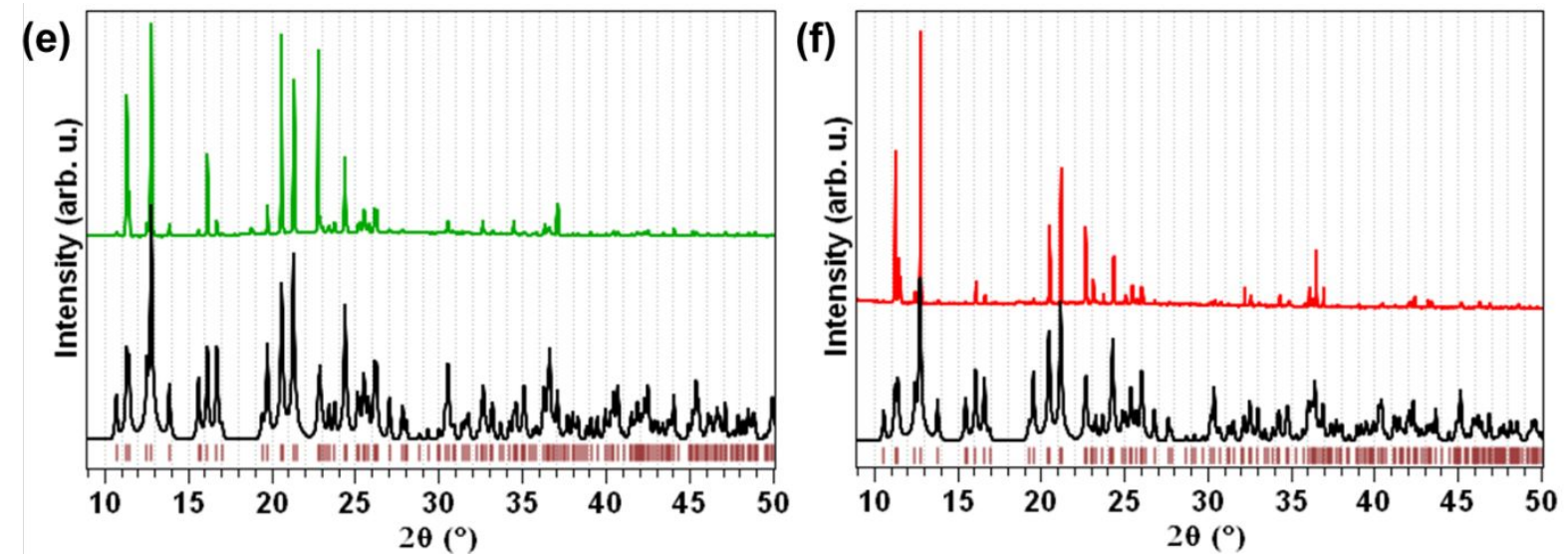

Figure S3. Comparison of the experimental powder X-ray diffraction pattern (a) for 5 (green line) and (b) for 6 (red line) with the calculated pattern from single crystals X-ray data (black line). The brown vertical lines indicate the position of the calculated diffraction lines. 

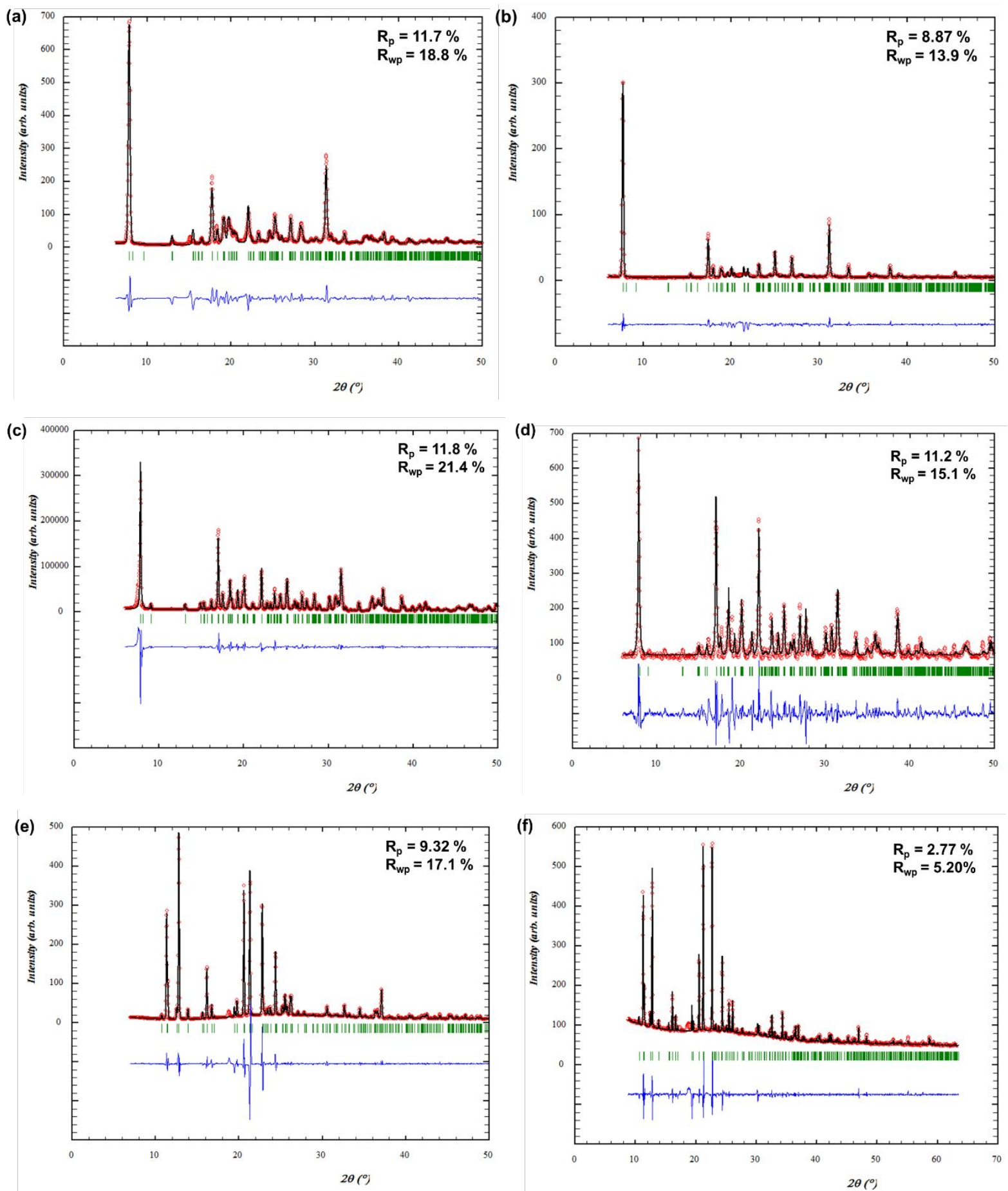

Figure S4. Observed (red), Lebail calculated (black) and difference (blue) XRPD patterns for compounds (a) 1, (b) 2, (c) 3, (d) 4, (e) 5 and (f) 6. The vertical bars indicate the Bragg positions. 


\section{SEM analysis}
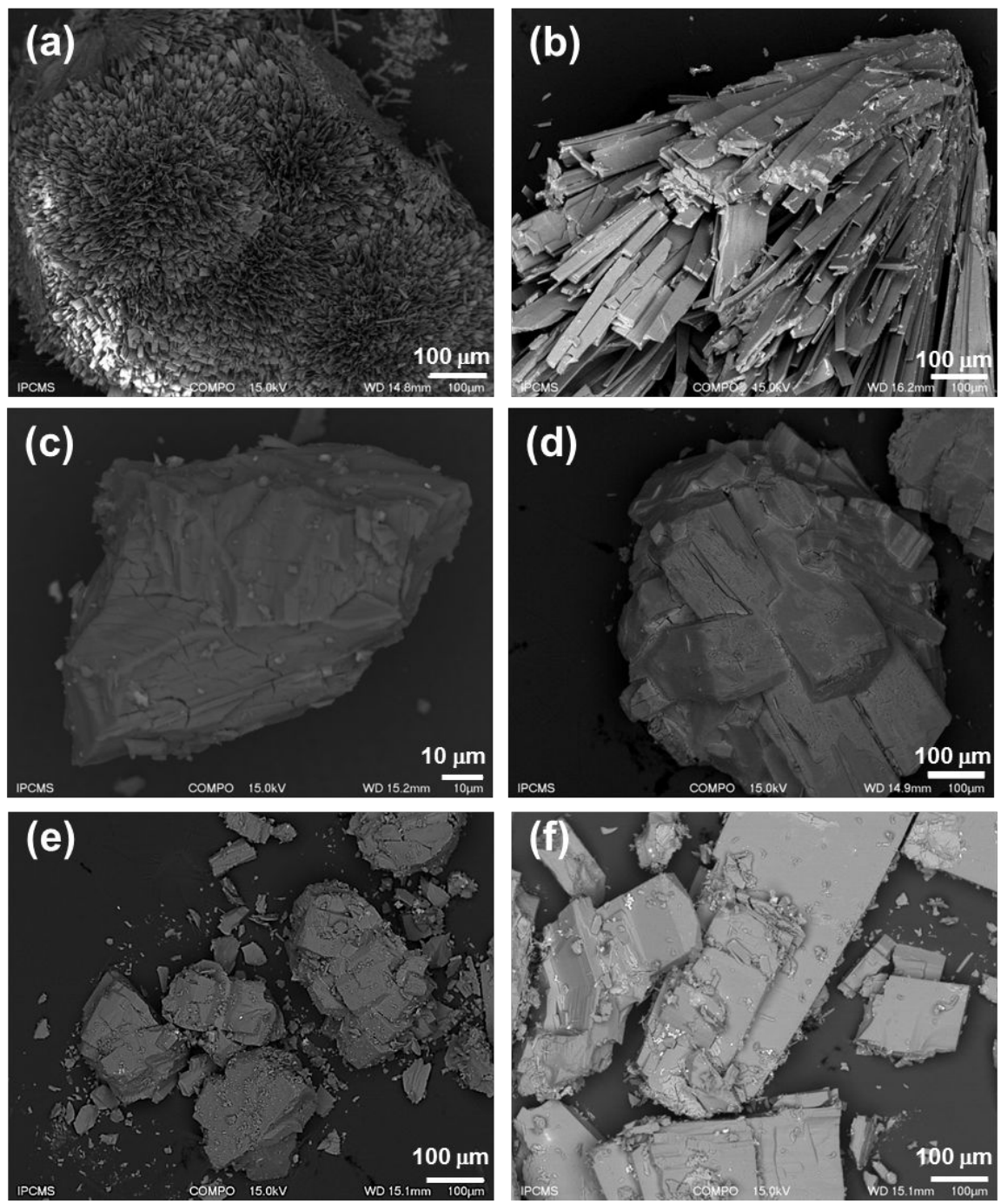

Figure S5. Representative SEM images in composition of the compounds (a) 1, (b) 2, (c) 3, (d) 4, (e) 5 and (f) 6 .

\section{Infrared analysis}

The infrared spectra of the six compounds are shown in Figure S6. The infrared spectrum of the imidazolium salt under its saline form $[\mathbf{L K}]$ is given for comparison.

The infrared spectra of $\mathbf{1}$ and $\mathbf{2}$ are very similar. They show a large band around $3400 \mathrm{~cm}^{-1}$ coming from hydrogen-bond involving water molecules. The vibration bands from the aromatic $\mathrm{C}-\mathrm{H}$ are visible at 3144 and $3090 \mathrm{~cm}^{-1}$ while those coming from the aliphatic $\mathrm{C}-\mathrm{H}$ are visible at 3067, 3006 and $2960 \mathrm{~cm}^{-1}$. Moreover, two bands are observed at $1566 \mathrm{~cm}^{-1}$ and at $1384 \mathrm{~cm}^{-1}$ assigned to the asymmetric $\left(v_{\mathrm{asym}}\right)$ and symmetric $\left(v_{\mathrm{sym}}\right)$ vibration of the carboxylate function, respectively. The difference $\Delta v\left(\Delta v=v_{\text {asym- }} v_{\text {sym }}\right)$ equal to $182 \mathrm{~cm}^{-1}$ is lower than the $\Delta v$ value 
of $[\mathbf{L K}]\left(\Delta v=236 \mathrm{~cm}^{-1}\right)$. These spectral features indicate that the carboxylate functions are in a bidentate coordination mode between one $\mathrm{Co}^{2+}$ and one $\mathrm{H}$ atom of the water molecule which is in good agreement with the crystal structure.

The infrared spectra of compounds 3-4 are rather similar to those of $\mathbf{1}$ in the range between $1700 \mathrm{~cm}^{-1}$ and $400 \mathrm{~cm}^{-1}$. Some differences can be observed between $2900 \mathrm{~cm}^{-1}$ and $3700 \mathrm{~cm}^{-1}$ since free water molecules are replaced by ethylene glycol molecules.

The infrared spectra of 5 and $\mathbf{6}$ are identical. They show a large band around $3370 \mathrm{~cm}^{-1}$ from the water molecule. The bands located at 3163 and $3100 \mathrm{~cm}^{-1}$ correspond to the aromatic $\mathrm{C}-\mathrm{H}$ while those at 3008,2985 and $2955 \mathrm{~cm}^{-1}$ to the aliphatic $\mathrm{C}-\mathrm{H}$ vibrations. The band at $1666 \mathrm{~cm}^{-1}$ is attributed to the bending vibration of the water molecule. Three asymmetric vibration bands coming from the $\mathrm{C}=\mathrm{O}$ bonds can be observed at 1632,1603 and $1583 \mathrm{~cm}^{-1}$, the latter coming from the oxalate ligand while the two formers are ascribed to the carboxylate functions of the imidazolium ligand. The symmetric vibration band of the C-O moieties is located at $1394 \mathrm{~cm}^{-}$ ${ }^{1}$. Based on the two different positions of the asymmetric vibration bands of the carboxylate functions, it is possible to determine two values of $\Delta v$ indicating various mode of coordination of the carboxylate functions. The first one is equal to $209 \mathrm{~cm}^{-1}$ and corresponds to a bridging bidentate coordination mode between two metals while the second, equal to $189 \mathrm{~cm}^{-1}$, corresponds to a monodentate coordination mode between one $\mathrm{Co}^{2+}$ and one $\mathrm{H}$ coming from the water molecule.

All features in the FTIR powder spectra are consistent with the single crystal structure.

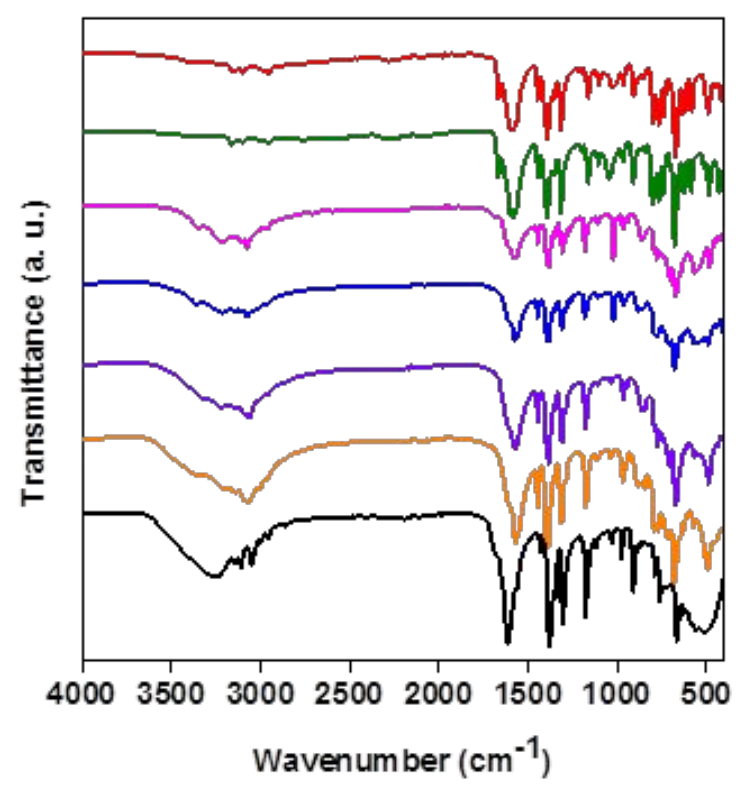

Figure S6. Infrared spectra of the compounds [LK] (black line), 1 (orange line), 2 (purple line), 3 (blue line), 4 (pink line), 5 (green line) and $\mathbf{6}$ (red line). 


\section{Thermal analysis}

The thermal stability of the six compounds has been studied by thermal analysis (Figure S7). The experiments were performed under air with a heating rate of $5^{\circ} \mathrm{C} / \mathrm{min}$.

(a)
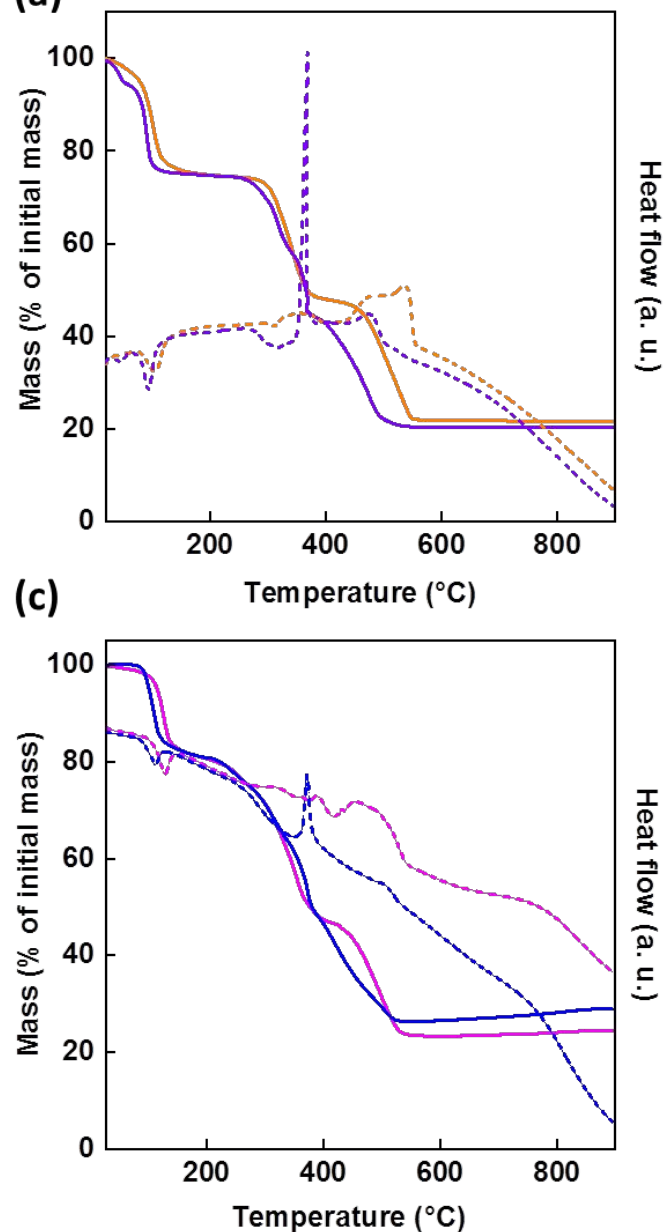

(b)

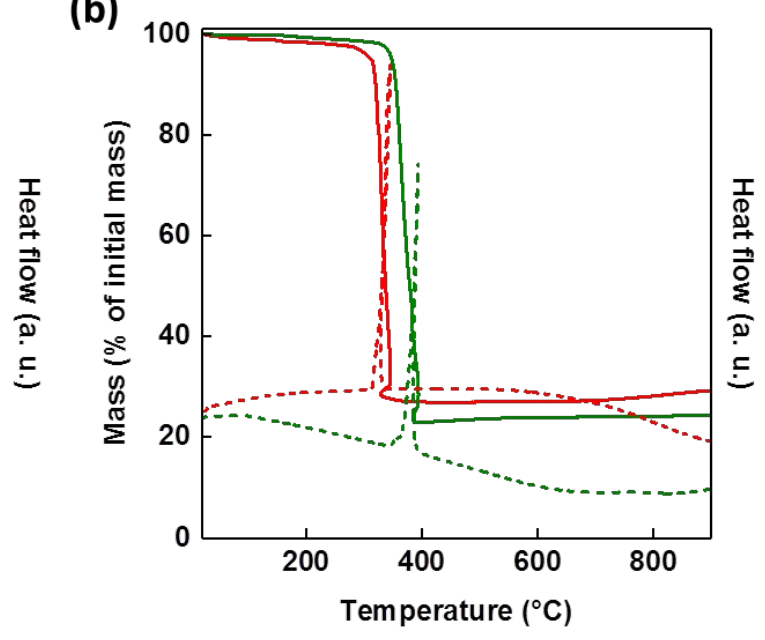

Figure S7. TGA (plain line) and TDA (dotted line) curves of the compounds (a) 1 (orange line) and $\mathbf{2}$ (purple line), (b) 3 (green line) and 4 (red line), (c) 5 (pink line) and 6 (blue line).

For the compound 1 , the first endothermic weight loss between $20^{\circ} \mathrm{C}$ and $195^{\circ} \mathrm{C}$ corresponds to the departure of the uncoordinated and the four coordinated water molecules (obsd. $25.23 \%$, calcd. $24.51 \%$ ). The two successive weight losses between $195^{\circ} \mathrm{C}$ and $600^{\circ} \mathrm{C}$, associated with exothermic peaks, correspond to the decomposition of the organic part, the removal of the free chloride anion and to the formation of nickel oxide $\mathrm{NiO}$ as identified by powder $\mathrm{X}$-ray diffraction (obsd. $54.53 \%$, calcd. $55.14 \%$ ).

The TGA of $\mathbf{2}$ is nearly the same except that the departure of uncoordinated and coordinated water molecules occurs in two distinct steps. Thus, the first endothermic weight loss between 
$20^{\circ} \mathrm{C}$ and $60^{\circ} \mathrm{C}$ is ascribed to the departure of the uncoordinated water molecule (obsd. 5.00 $\%$, calcd. $4.90 \%$ ) while the second between $60^{\circ} \mathrm{C}$ and $190^{\circ} \mathrm{C}$ is ascribed to the departure of the four coordinated water molecules (obsd. $19.41 \%$, calcd. $19.60 \%$ ). The two last exothermic weight losses at higher temperature correspond to the decomposition of the organic part, the removal of the free chloride anion and to the formation of cobalt oxide $\mathrm{Co}_{3} \mathrm{O}_{4}$ as identified by powder X-ray diffraction of the residue (obsd. $54.42 \%$, calcd. $53.67 \%$ ).

For the compound 3 and 4 , the first endothermic peak between $20^{\circ} \mathrm{C}$ and $190^{\circ}$ Care ascribed to the departure of the four water molecules (obsd. $19.10 \%$, calcd. $18.94 \%$ for 3 and obsd. $19.10 \%$, calcd $18.92 \%$ for 4 ). Then, the two successive exothermic weight losses between $190^{\circ} \mathrm{C}$ and $600^{\circ} \mathrm{C}$ are associated to the removal of the ethyleneglycol, the decomposition of the organic ligand and the formation of $\mathrm{NiO}$ or $\mathrm{Co}_{3} \mathrm{O}_{4}$ as identified by powder $\mathrm{X}$-ray diffraction of the residue (obsd. $60.33 \%$, calcd. $61.68 \%$ for 3 and obsd. $57.81 \%$, calcd $60.25 \%$ for 4 ).

The TGA curves of $\mathbf{5}$ and $\mathbf{6}$ are simpler since there is only one exothermic weight loss between $200^{\circ} \mathrm{C}$ and $400^{\circ} \mathrm{C}$ associated with the elimination of the bridging water molecule, the organic part (i. e. the oxalate ligand and the [L]- ligand) and the concomitant formation of cobalt oxide $\mathrm{Co}_{3} \mathrm{O}_{4}$ or nickel oxide $\mathrm{NiO}$, respectively, as identified by powder X-ray diffraction of the residue (obsd. $73.18 \%$, calcd. $73.87 \%$ for 5 and obsd. $74.95 \%$, calcd. $75.28 \%$ for $\mathbf{6}$ ).

All these data underline the homogeneity of the different compounds.

\section{UV-Visible-NIR spectroscopy}

The six compounds have been characterized by UV-Visible-NIR spectroscopy in the solid state. The compounds $\mathbf{1}, \mathbf{3}$ and $\mathbf{5}$ show the characteristic transitions of $\mathrm{Ni}^{2+}$ ions in octahedral environment (see Figure S8a and Table S5). The compounds 2, 4 and 6 show the characteristic transitions of $\mathrm{Co}^{2+}$ ions in octahedral environment in a high spin configuration (see Figure S8b and Table S6). 
(a)

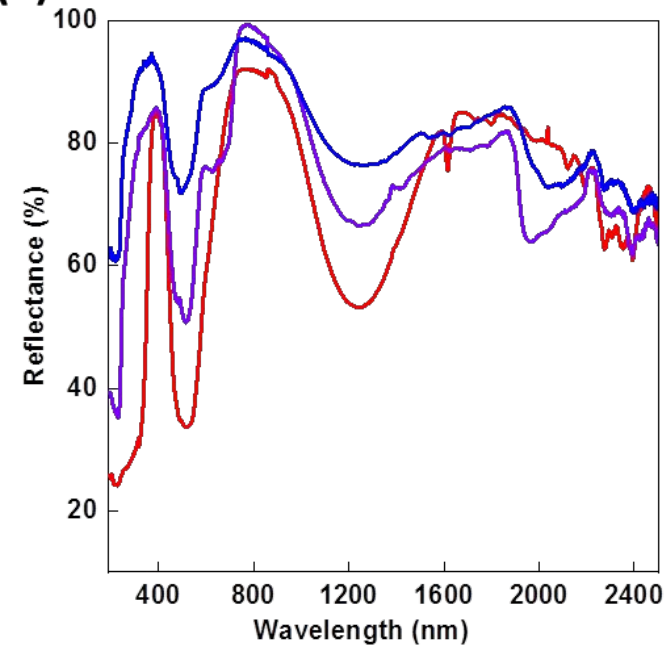

(b)

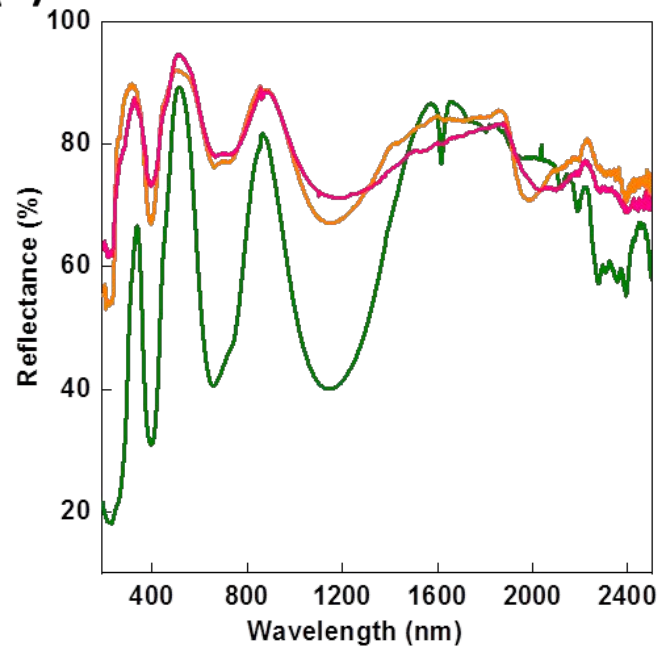

Figure S8. Solid state UV-Visible-NIR spectra of the compounds (a) 1 (orange line), $\mathbf{3}$ (pink line), 5 (green line) and (b) 2 (purple line), 4 (blue line) and $\mathbf{6}$ (red line).

Table S5. Bands assignment and crystal field parameters ${ }^{1}$ for $\mathbf{1}, \mathbf{2}$ and $\mathbf{3 .}$

\begin{tabular}{cccccccc}
\hline & $\begin{array}{c}\lambda_{1} \\
(\mathbf{n m})\end{array}$ & $\begin{array}{c}\lambda_{2} \\
(\mathbf{n m})\end{array}$ & $\begin{array}{c}\lambda_{3} \\
(\mathbf{n m})\end{array}$ & $\begin{array}{c}\mathbf{D q} \\
\left(\mathbf{c m}^{-1}\right)\end{array}$ & $\begin{array}{c}\mathbf{B} \\
\left(\mathbf{c m}^{-1}\right)\end{array}$ & $\mathbf{D q} / \mathbf{B}$ & $\boldsymbol{\beta}$ \\
\hline$\left[\mathbf{N i}(\mathbf{L})\left(\mathbf{H}_{\mathbf{2}} \mathbf{O}\right)_{4}\right][\mathbf{C l}] . \mathbf{H}_{\mathbf{2}} \mathbf{O}(\mathbf{1})$ & 396 & 664 & 1148 & 871 & 883 & 0.99 & 0.82 \\
$\left.\left[\mathbf{N i}(\mathbf{L})\left(\mathbf{H}_{\mathbf{2}} \mathbf{O}\right)_{4}\right][\mathbf{C l}] . \mathbf{( E G}\right)_{\mathbf{0 . 5}} \mathbf{( 3 )}$ & 397 & 664 & 1178 & 849 & 922 & 0.92 & 0.85 \\
{$\left[\mathbf{N i}(\mathbf{L})(\mathbf{0 x})_{\mathbf{0 . 5}}\left(\boldsymbol{\mu}_{\mathbf{2}}-\mathbf{H}_{\mathbf{2}} \mathbf{O}\right)_{\mathbf{0 . 5}} \mathbf{( 5 )}\right.$} & 396 & 662 & 1144 & 874 & 908 & 0.96 & 0.84 \\
\hline
\end{tabular}

N.B.: $\lambda_{1}, \lambda_{2}, \lambda_{3}$ corresponds to the transitions ${ }^{3} A_{2} g(F) \rightarrow{ }^{3} T_{1 g}(P),{ }^{3} A_{2 g}(F) \rightarrow{ }^{3} T_{l g}(F)$ and ${ }^{3} A_{2 g}(F) \rightarrow$ ${ }^{3} T_{2 g}(F)$ of octahedral $\mathrm{Ni}^{2+}$, respectively. ${ }^{2,3}$

Table S6. Bands assignment and crystal field parameters ${ }^{1}$ for $\mathbf{2 , 4}$ and $\mathbf{6}$.

\begin{tabular}{cccccccccc}
\hline & $\begin{array}{c}\lambda_{1} \\
(\mathbf{n m})\end{array}$ & $\begin{array}{c}\lambda_{2} \\
(\mathbf{n m})\end{array}$ & $\begin{array}{c}\lambda_{3} \\
(\mathbf{n m})\end{array}$ & $\begin{array}{c}\mathbf{D q} \\
\left(\mathbf{c m}^{-1}\right)\end{array}$ & $\begin{array}{c}\text { B } \\
\left(\mathbf{c m}^{-1}\right)\end{array}$ & $\mathbf{D q} / \mathbf{B}$ & $\boldsymbol{\beta}$ & $\mathbf{c}$ & $\mathrm{A}$ \\
\hline$\left[\mathbf{C o}(\mathbf{L})\left(\mathbf{H}_{\mathbf{2}} \mathbf{O}\right)_{4}\right][\mathbf{C l}] . \mathbf{H}_{\mathbf{2}} \mathbf{O}(\mathbf{2})$ & 515 & 625 & 1257 & 907 & 838 & 1.08 & 0.75 & 0.19 & 1.4 \\
{$\left[\mathbf{C o}(\mathbf{L})\left(\mathbf{H}_{\mathbf{2}} \mathbf{O}\right)_{4}\right][\mathbf{C l}] .(\mathbf{E G})_{\mathbf{0 . 5}}(\mathbf{4})$} & 498 & 624 & 1255 & 911 & 883 & 1.03 & 0.79 & 0.19 & 1.4 \\
{$\left[\mathbf{C o}(\mathbf{L})(\mathbf{0 x})_{\mathbf{0 . 5}}\left(\boldsymbol{\mu}_{\mathbf{2}}-\mathbf{H}_{\mathbf{2}} \mathbf{O}\right)_{\mathbf{0 . 5}}\right](\mathbf{6})$} & 520 & - & 1246 & 913 & 821 & 1.11 & 0.73 & & 1.4 \\
\hline
\end{tabular}

N.B.: $\lambda_{1}, \lambda_{2}, \lambda_{3}$ corresponds to the transitions ${ }^{4} T_{l g}(F) \rightarrow{ }^{4} T_{l g}(P),{ }^{4} T_{l g}(F) \rightarrow{ }^{4} A_{2 g}(F)$ and ${ }^{4} T_{l g}(F) \rightarrow{ }^{4} T_{2 g}(F)$ of octahedral $\mathrm{Co}^{2+}$, respectively. ${ }^{2,4}$

Using the transitions observed corresponding to octahedral $\mathrm{Co}^{2+}$ species, it was possible to determine the constant $A$ with the following expression: ${ }^{5}$ 
$\mathrm{A}=\frac{3 / 2-\mathrm{c}^{2}}{1+c}$

where $\mathrm{c}=0.75+1.875 \frac{\mathrm{B}}{\mathrm{Dq}}-1.25\left[1+1.8 \frac{\mathrm{B}}{\mathrm{Dq}}+\left(\frac{\mathrm{B}}{\mathrm{Dq}}\right)^{2}\right]^{1 / 2}$ 


\section{Magnetic data}
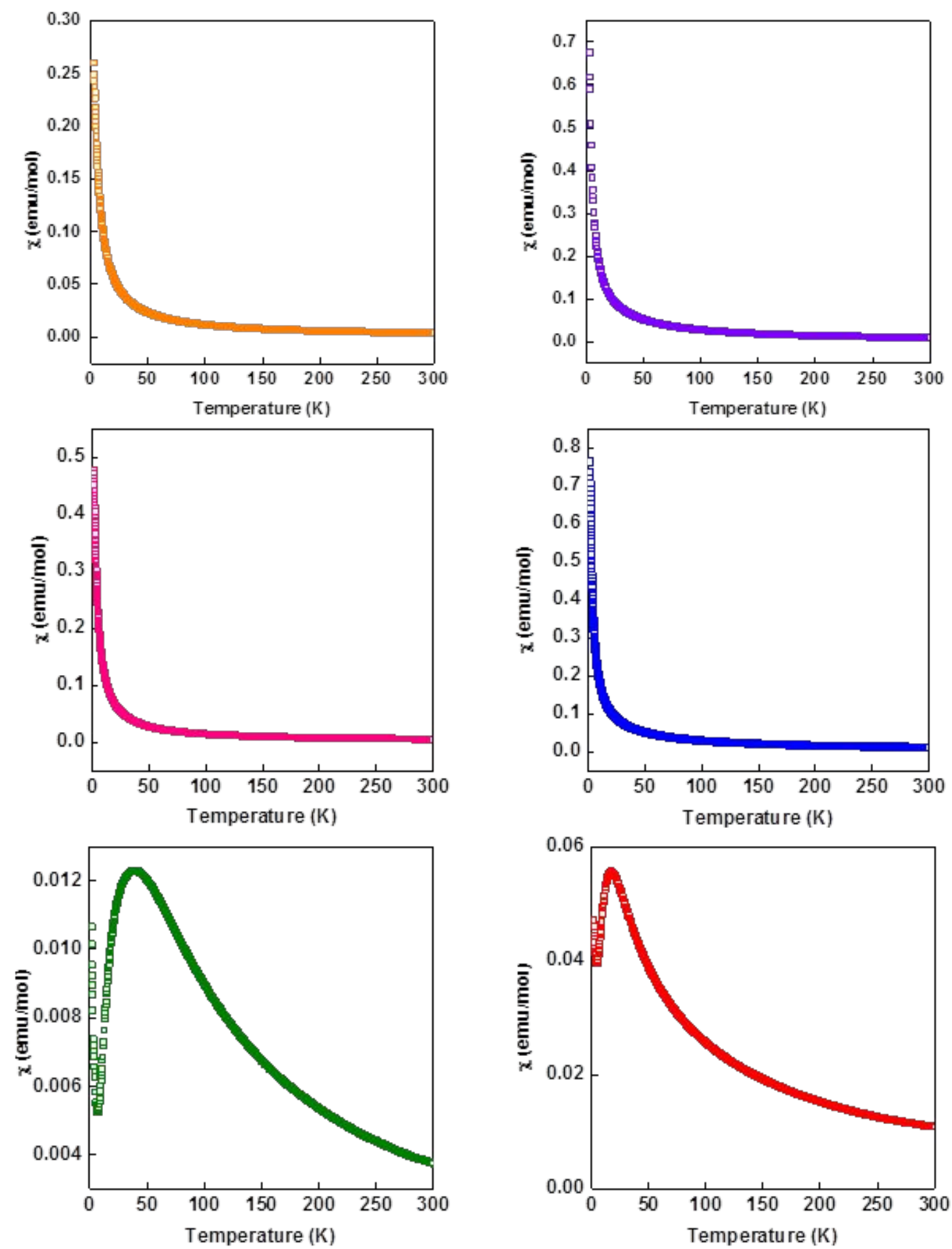

Figure S9. Temperature variation of the magnetic susceptibility at an applied field of $5000 \mathrm{G}$ between 1.8 and $300 \mathrm{~K}$ for the compounds $\mathbf{1}$ (orange) and $\mathbf{2}$ (purple), $\mathbf{3}$ (pink) and $\mathbf{4}$ (blue), $\mathbf{5}$ (green), 6 (red). 

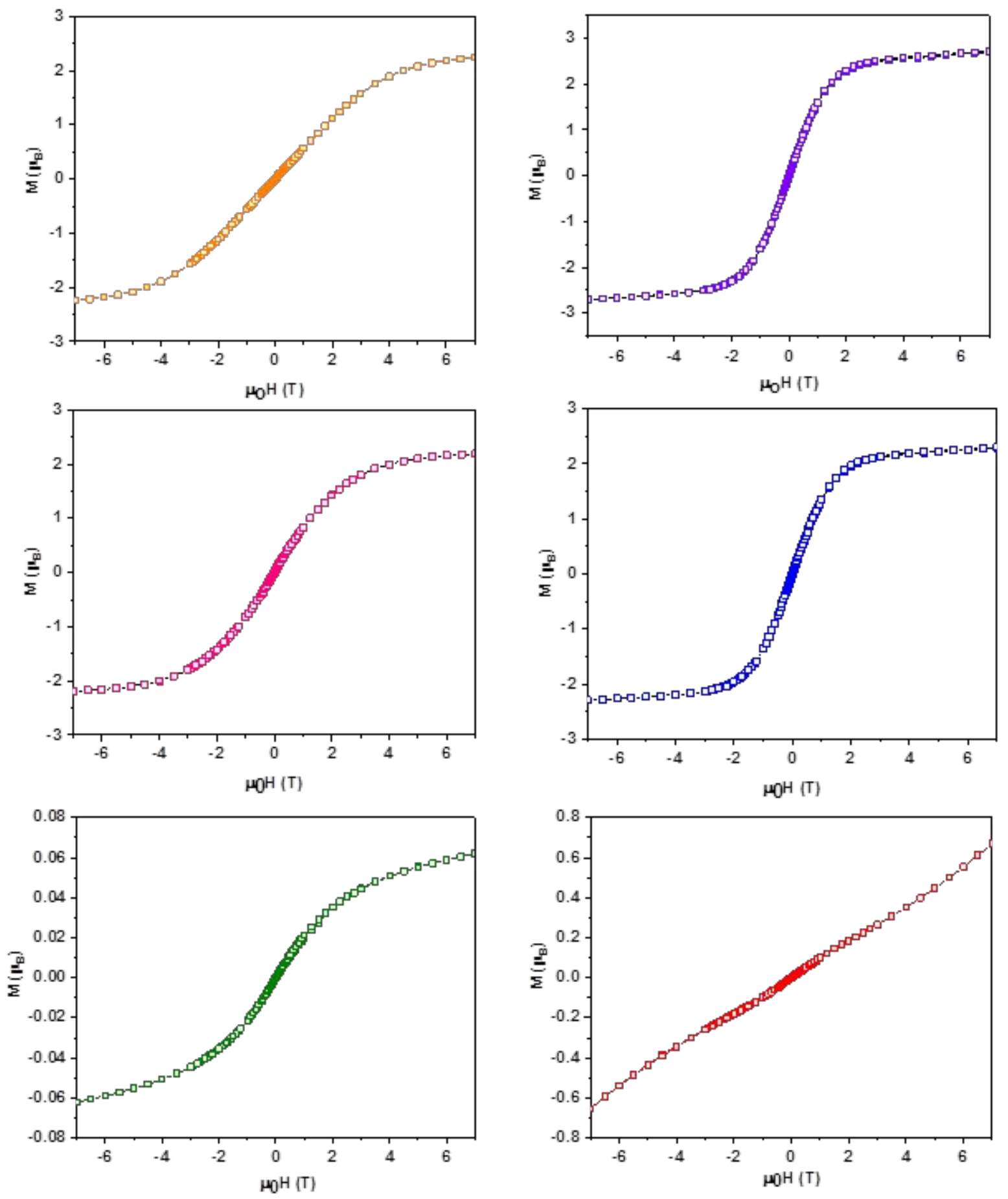

Figure S10. Magnetization versus field curve at $1.8 \mathrm{~K}$ for the compounds 1 (orange) and 2 (purple), $\mathbf{3}$ (pink) and $\mathbf{4}$ (blue), $\mathbf{5}$ (green), 6 (red). 


\section{Expression E1}

$\hat{H}=\sum_{1}^{\infty}\left(U_{1} \hat{S}_{i} \hat{S}_{i+1}+J_{2} \hat{S}_{i+1} \hat{S}_{i+2}\right)-g \mu_{B} \sum_{1}^{\infty} \hat{S}_{i} H$

Where the first term corresponds to the exchange Hamiltonian and the second to the Zeeman interaction.

\section{References}

(1) Dou, Y. Equations for Calculating Dq and B. J. Chem. Educ. 1990, 67, 134.

(2) Lever, A. B. P. Inorganic Electronic Spectroscopy; Elsevier, 1984.

(3) Demessence, A.; Mesbah, A.; François, M.; Rogez, G.; Rabu, P. Structure and Magnetic Properties of a New 1D Nickel(II) Hydroxythiophenedicarboxylate. Eur. J. Inorg. Chem. 2009, 2009, 3713-3720.

(4) Delahaye, E.; Moulin, R.; Aouadi, M.; Trannoy, V.; Beaunier, P.; Fornasieri, G.; Bleuzen, A. Co2+@Mesoporous Silica Monoliths: Tailor-Made Nanoreactors for Confined Soft Chemistry. Chem. - Eur. J. 2015, 21, 16906-16916.

(5) Lloret, F.; Julve, M.; Cano, J.; Ruiz-García, R.; Pardo, E. Magnetic Properties of SixCoordinated High-Spin Cobalt(II) Complexes: Theoretical Background and Its Application. Inorganica Chim. Acta 2008, 361, 3432-3445. 